\title{
Monitoring of large structure using distributed Brillouin fibre sensing
}

\author{
Luc Thévenaz, Massimo Facchini, Alexandre Fellay, Philippe Robert \\ EPFL, Swiss Federal Institute of Technology, Metrology Lab, CH-1015 Lausanne, Switzerland \\ Daniele Inaudi*, Boris Dardel ${ }^{* *}$ \\ * Smartec SA, Via al Molino 6, CH-6916 Grancia, Switzerland \\ ** Alcatel Cable Suisse SA, CH-2016 Cortaillod, Switzerland
}

\begin{abstract}
On-site distributed measurements using a sensor based on stimulated Brillouin scattering are presented. Long fibre length can be used, so that a dense 2D or 3D measurement of strain or temperature can be obtained in large structure.
\end{abstract}

Optical fibre sensors based on stimulated Brillouin scattering have now clearly demonstrated their excellent capability for long-range distributed strain and temperature measurements ${ }^{1,2,3}$. The Brillouin interaction causes the coupling between optical and acoustical waves when a resonance condition is fulfilled. It turns out that this resonance condition is strain and temperature-dependent, so that determining the resonance frequency directly provides a measure of temperature or strain

The resonance frequency is an intrinsic property of the material that may be observed in any silica fibre. This is very attractive since the bare fibre itself acts as sensing element without any special fibre processing or preparation. Standard optical cables may thus be used, resulting in a low-cost sensing element that may be left in the structure. Since the optical effect only depends on the fibre material, it is absolutely stable in time and independent of the instrument. Different measurements performed over a long-term period are thus fully comparable.

Instead of using the now traditional configuration using two laser sources, ${ }^{1,2}$ a novel experimental configuration has been developed by our team, ${ }^{3,4,5}$ shown in Fig. 1.

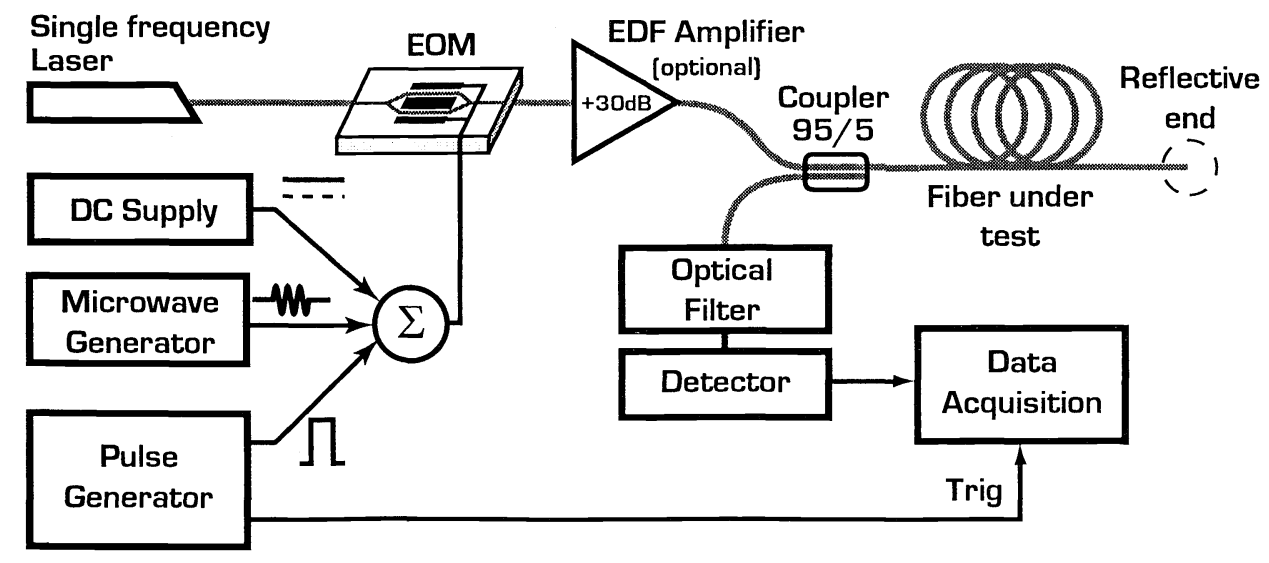

Fig. 1 Experimental set-up for distributed Brillouin gain spectrum measurements, using an electro-optic modulator to generate the interacting signals. 
Its main original feature is the presence of a single laser source that is modulated through a MachZehnder electro-optic modulator (EOM). This electro-optic modulator is the key element of the set-up since it is used on the one hand for pulsing the CW light from a single frequency laser to form the pump signal, and on the other hand for the generation and frequency tuning of the probe signal. The frequency shift on the laser light is achieved by simply applying a microwave signal on the electro-optic modulator electrodes, that creates a sideband at the proper frequency in the laser spectrum. This gives to the system an inherent stability, as far as frequency drifts of the laser are concerned.

The spatial resolution obtained with this equipment is 1 meter for a $10 \mathrm{~km}$ range. The physical limit for spatial resolution, that is just below 1 meter and results from the acoustic properties of silica, is actually reached by the equipment for short measurement range $(<1 \mathrm{~km})$. This kind of sensors is thus definitely dedicated for long range measurements with meter resolution and is not suitable for centimeter resolution. The accuracy on the determination of the Brillouin shift $v_{B}$ depends on the amplification contrast and the probe signal intensity. In standard fibres an accuracy of $1 \mathrm{MHz}$ is observed. This approximately corresponds to a $1 \mathrm{~K}$ temperature resolution and to a $2 \times 10^{-5}$ strain resolution. The Brillouin shift accuracy can be improved to $250 \mathrm{kHz}$, corresponding to a $0.25 \mathrm{degC}$ temperature and $5 \times 10^{-6}$ strain resolutions, respectively, at the expense of a worse spatial resolution or a longer measurement time.

\section{Strain measurement}

The sensor has been used in the field in various situations, such as the monitoring of the strain experienced by a fibre telecommunication cable as a result of the installation or even the check of the local resistance of a marine rope.

To demonstrate the capability of the instrument to perform truly continuous distributed measurements, the bending of a $12 \mathrm{~m}$ aluminium beam under its own weight was monitored. The strain experienced by the beam is proportional to the curvature and follows a parabolic law. The fibre was fixed along the Ushaped beam using glue. Fig. 2 shows the measurements performed in two situations, for a central and an end beam fixing point. The agreement with the theoretical strain distribution is excellent, taking into account the small amplitude of strain the uncertainties to be expected in such an experiment.
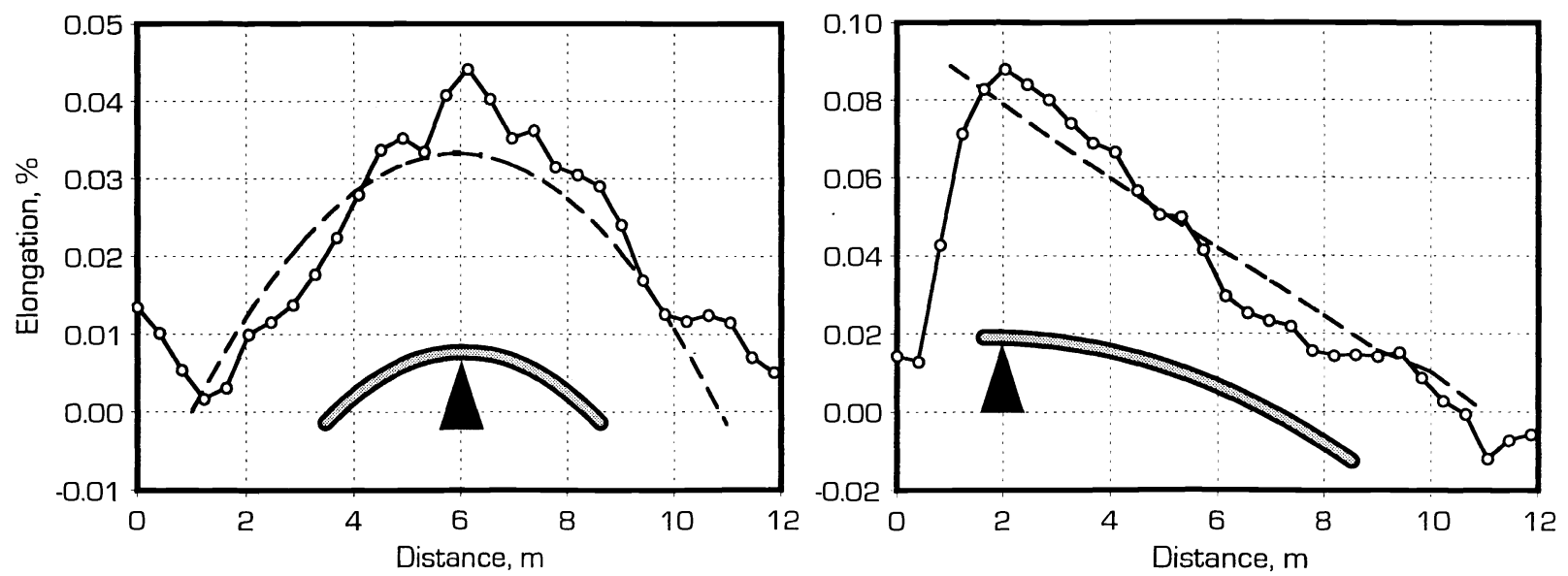

Fig. 2 Strain experienced by a $12 \mathrm{~m}$ U-shaped aluminium beam bent by its own weight in two different experimental configurations. The solid curves show the measurements performed by the optical fibre Brillouin sensor and the dashed curves represents the calculated expected strain. 


\section{Temperature measurement}

Civil engineering is an important field of application for distributed Brillouin sensing, since it perfectly fulfils the requirement of long range measurements with meter spatial resolution. The equipment was used to monitor the concrete setting temperature in large structures. This monitoring is of prime importance in critical works, since the density and the importance of microcracks are directly related to the

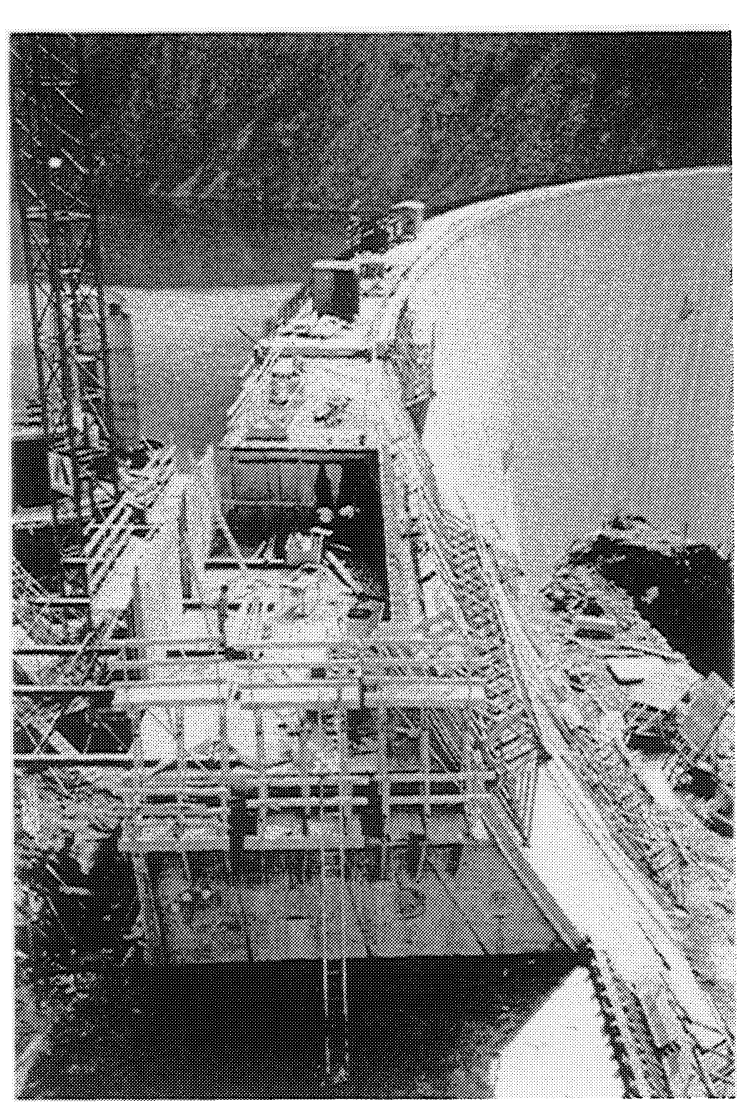
maximum temperature experienced by the concrete during the setting chemical process.

A major dam at Luzzone in the Swiss Alps was recently raised to increase the power capability of the associated hydroelectric plant. This raising was actually achieved by stacking gradually new concrete slabs of $15 \mathrm{~m} \times 10 \mathrm{~m}$ average size for a $3 \mathrm{~m}$ thickness, as shown in Fig. 3. A small optical telecommunication cable was installed during the concrete pouring over the central layer of the largest slab, so that the embedded cable makes a dense horizontal mat, necessary to obtain a twodimensional temperature distribution of the whole central slab area. Fig. 4 shows the temperature distribution over the slab at different moment after concreting. It can be clearly seen that the temperature rises up to $50 \operatorname{deg} C$ in the central area and that it takes many weeks to cool down this region. The outer slab areas rapidly stabilise at the ambient temperature, so that an observer is totally unaware that the concrete is still fairly hot in the central region of the dam.

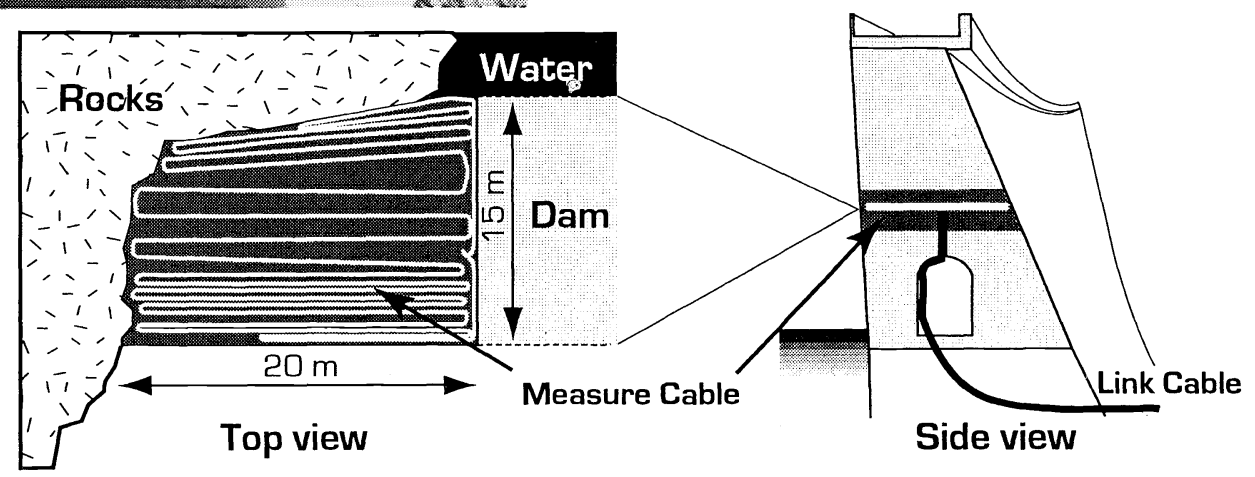

Fig 3 View of the concrete slab and of the mat-like installation of the measuring cable for concrete setting temperature monitoring, during the raising of Luzzone dam in the Swiss Alps.

\section{Conclusion}

Demonstrative tests of distributed temperature and strain measurements have been performed in the field using an instrument based on the local analysis of the stimulated Brillouin interaction (LASBI). A problem that is often pointed out is the impossibility to discriminate between temperature and strain effect 


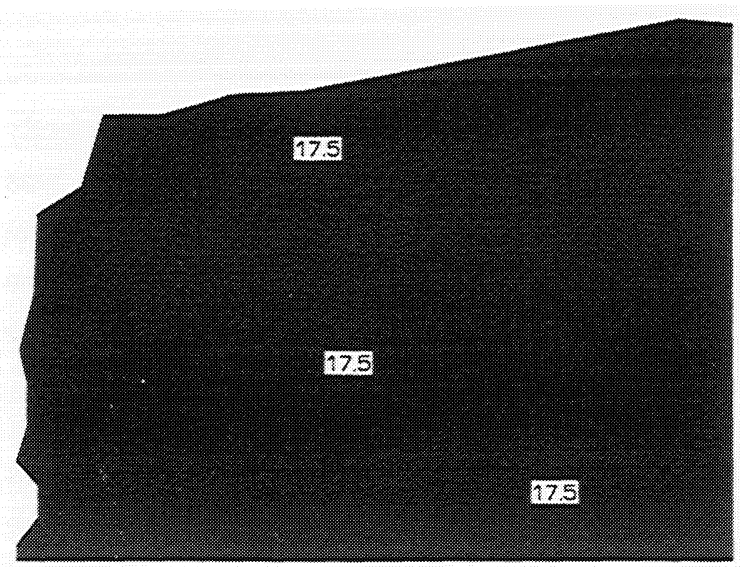

After concreting

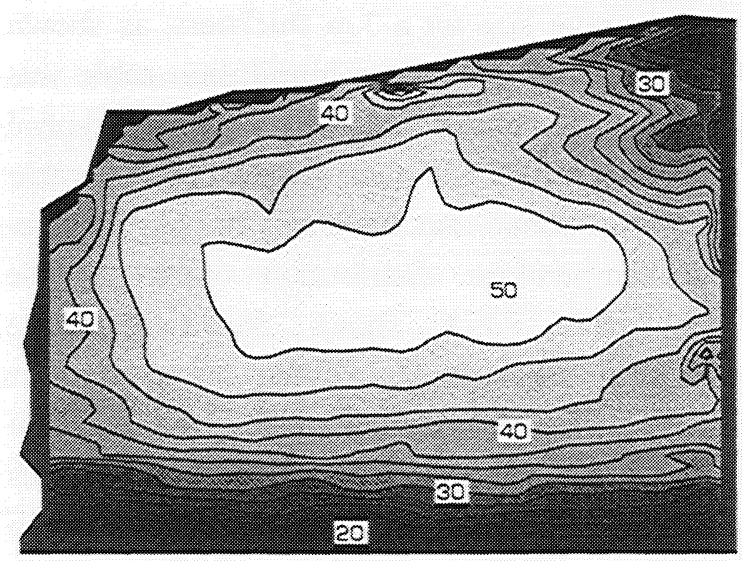

30 days later

Fig. 4 Two-dimensional temperature distribution in Luzzone dam during the setting of the concrete slab shown in Fig. 3, obtained by the distributed Brillouin sensor using an optical cable embedded within the concrete.

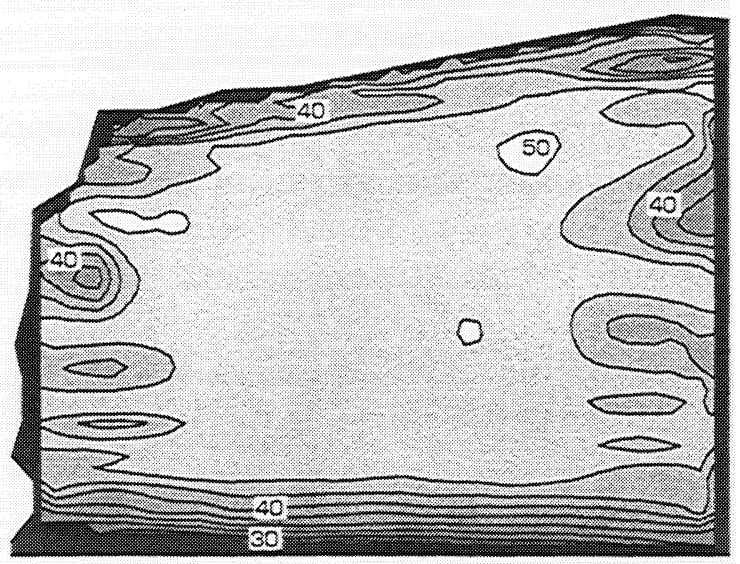

5 days later

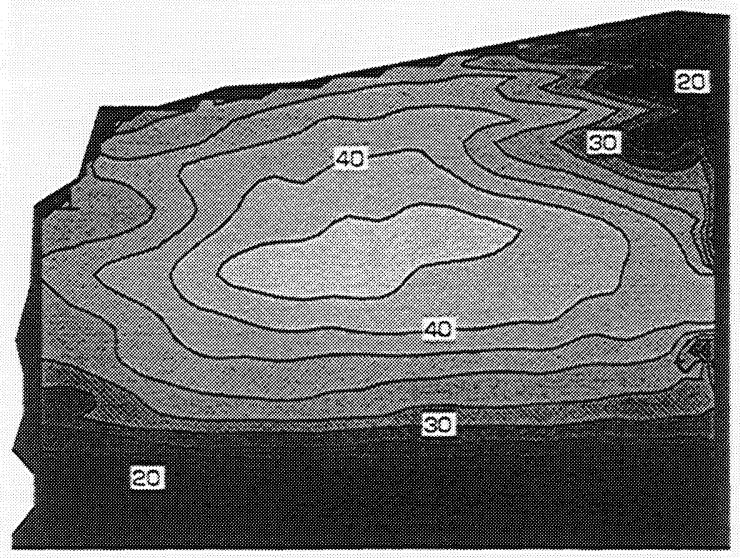

55 days later

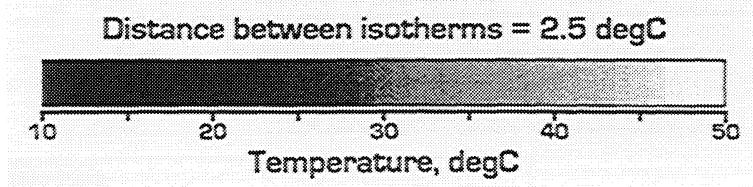

with a Brillouin shift measurement. The problem can be in real conditions easily worked out by combining loose-tubed and tight cables. In a loose tube the fibre is granted to be unstrained and is therefore only sensitive to temperature. If strain information must be obtained a parallel tight cable must be installed that is fixed on the structure, so that the fibre can experience strain. The drawback of a double-length sensor is widely canceled out by the very long range capability of such a sensor. This double-length configuration is an effective way to grant a maintained accuracy for both temperature and strain.

\section{References}

1. T.Horiguchi, T.Kurashima, M.Tateda, Photonics Tech. Lett., 2, p. 352, 1990.

2. X. Bao, J. Dhliwayo, N. Heron, D.J. Webb, D.A. Jackson, J. Lightwave Technol., 13, p. 1340, 1995.

3. M.Niklès, L.Thévenaz, P.Robert, Optics Lett., 21, pp. 758-760, 1995.

4. M.Niklès, L.Thévenaz, Ph.Robert, J. Lightwave Technol., LT-15, pp. 1842-1851, 1997.

5. L.Thévenaz, M.Niklès, A.Fellay, M.Facchini, Ph.Robert, SPIE Proceedings vol.3330, pp.301-314, 1998. 